\title{
Effects of Xylooligosaccharides on Blood Glucose, Serum and Liver Lipids and Cecum Short-chain Fatty Acids in Diabetic Rats
}

\author{
Katsumi Imaizumi, Yoriko Nakatsu, Masao Sato, \\ Yasni Sedarnawati and Michihiro Sugano \\ Laboratory of Nutrition Chemistry, Department of Food Science \\ and Technology, School of Agriculture (46-09), \\ Kyushu University, Fukuoka 812, Japan
}

Received August 9, 1990

\begin{abstract}
The effect of supplementing xylooligosaccharides $(\mathrm{XO})$ to purified diets on growth performance and several metabolic parameters was examined in streptozotocin-induced diabetic rats. The dietary XO improved growth retardation, hyperphagia, polydipsia, elevation of serum glucose, triglyceride and cholesterol, reduction of liver triglyceride, and fatty acid composition (reduction of the desaturation index) of liver phosphatidylcholine. In addition, the XO diet yielded an acidic environment in the cecum by increasing the pool size of acetic acid. Thus, $\mathrm{XO}$, which is half as sweet as sucrose, can be applicable to foods as a sweetener that is capable of improving diabetic symptoms.
\end{abstract}

The diverse beneficial effects on human health of polysaccharides that are resistant to digestion are now becoming recognized so widely that an application of these polysaccharides to chronic diseases such as ischemic heart disease, ${ }^{1)}$ diabetes mellitus, ${ }^{2)}$ diverticular disease $^{3)}$ and cancer ${ }^{4)}$ has been extensively advocated. Apart from the interest in these polysaccharides, oligosaccharides resistant to digestive enzymes have not drawn attention until recently, when some of them were produced on an industrial scale, and their physiological significance was revealed. At present, fructooligosaccharides, ${ }^{5)}$ galactooligosaccharides $^{6)}$ and lactulose ${ }^{7)}$ have been shown to stimulate specifically the growth of Bifidobacterium, which is believed to maintain preferably the colonic environment in humans. ${ }^{8)}$ In addition, fructooligosaccharides have been shown to exert beneficial effect on diabetic subjects by lowering the levels of blood sugar and lipids. ${ }^{9)}$ Lactulose has been utilized for lowering the blood ammonia concentration in patients with portosystemic encephalopathy. ${ }^{10}$ )

Unlike these hexose oligosaccharides just mentioned, new xylooligosaccharides (XO) developed from xylan by the action of xylobiose (4-D- $\beta$-D-xylopyranosyl- $\beta$-D-xylose) formation enzymes derived from bacteria are mainly composed of two and three xylose units with a $\beta-1-4$ linkage. ${ }^{11)} \mathrm{XO}$ is approximately half as sweet as sucrose and relatively stable in an acidic medium. ${ }^{11)}$ The latter property may endow protection from decomposition when passing through the stomach.

Although the distribution of $\mathrm{XO}$ in the natural world has not been extensively studied hitherto, xylobiose is relatively abundant in bamboo shoots, which have been traditionally consumed in Japan and China. ${ }^{11)}$ These characteristics of XO led us to examine an application as a sweetener to patients such as those with diabetes mellitus and obesity who are prohibited from taking large amounts of sucrose. The present study reports the effects of $\mathrm{XO}$ on several parameters concerning growth, feeding behavior, metabolism of glucose and lipids, and production of shortchain fatty acids as an indicator of the colonic environment in streptozotocin-induced diabetic rats. 


\section{Materials and Methods}

Experimental animals. Male Wistar rats, 7 weeks old, obtained from Clea Japan (Osaka) were raised in our animal facilities under a constant temperature $\left(23 \pm 1^{\circ} \mathrm{C}\right)$ and maintained dark-light cycle (lights on, 08:00-18:00). The rats were freely given water and commercial feed (Type NMF, Oriental Yeast Co., Tokyo) until the experimental diets later described were given. At eight weeks of age, the rats were treated with streptozotocin (Sigma Chemical Co., MO). The streptozotocin was freshly prepared in physiological saline and injected into the femoral vein at a dose of $60 \mathrm{mg} / \mathrm{kg}$ of body weight under diethyl ether anesthesia. ${ }^{12)}$ The body weight, and food and water intake were recorded every other day. Blood was taken from the tail vein between $11: 00-12: 00$ at the end of first and third weeks in the course of the experimental period. At the end of the fifth week, the rats were sacrificed by decapitation at 11:00-12:00, and blood serum was obtained by centrifugation. The liver and cecum were obtained immediately and frozen by liquid nitrogen until needed for analysis.

Diets. XO, which was prepared by hydrolyzing xylan with the xylobiose formation enzyme prepared from Trichoderma, ${ }^{11}$ was kindly supplied by Suntory Ltd., Osaka. The preparation of XO, composed of $23.5 \%$ xylose, $59.5 \%$ xylobiose, $12.9 \%$ xylotriose, $3.1 \%$ xylotetraose and $1.0 \%$ xylopentaose by weight, contained $15.7 \%(\mathrm{w} / \mathrm{w})$ water. The composition of the basal diet formulated according to formula $A$ of Oriental Yeast Co. (Tokyo) is shown in Table I. ${ }^{13}$ ) Ten $\%$ of XO was supplemented to the basal diet by replacing $5 \%$ each of corn starch and sucrose (Experiment 1) or 10\% of corn starch (Experiment 2 ) in the basal diet. The rats were raised on these diets for 5 weeks.

Table I. COMPOSITION OF THE DIETS

\begin{tabular}{|c|c|c|c|}
\hline \multirow{2}{*}{ Ingredient } & \multirow{2}{*}{$\begin{array}{c}\text { Basal } \\
\text { diet }\end{array}$} & \multicolumn{2}{|c|}{ XO diets } \\
\hline & & Exp. 1 & Exp. 2 \\
\hline $\begin{array}{l}\text { Milk casein } \\
\quad(\text { vitamin free })^{a}\end{array}$ & 25 & 25 & 25 \\
\hline Safflower oil ${ }^{b}$ & 6 & 6 & 6 \\
\hline Mineral $\operatorname{mix}^{a, c}$ & 6 & 6 & 6 \\
\hline Vitamin $\operatorname{mix}^{a, c}$ & 2 & 2 & 2 \\
\hline Cellulose $^{d}$ & 8 & 8 & 8 \\
\hline Corn starch ${ }^{a}$ & 38 & 33 & 28 \\
\hline$\alpha$-Corn $\operatorname{starch}^{a}$ & 10 & 10 & 10 \\
\hline Sucrose & 5 & 0 & 5 \\
\hline Xylooligosaccharide & 0 & 10 & 10 \\
\hline
\end{tabular}

a Obtained from Oriental Yeast Co., Tokyo.

b Linol Salada Oil Co., Tokyo.

Oriental mixture (Oriental Yeast Co,

d Toyo Roshi Co., Tokyo.
Analytical procedure. Commercially available kits were used to determine the glucose (Glucose Test, Wakq Pure Chemical Co., Osaka), cholesterol (Cholesterol C-test, Wako Pure Chemicals Co.) and triglyceride (Triglyceride G-test, Wako Pure Chemicals Co.) in the serum. Liver lipids were extracted according to the method of Folch et al. ${ }^{14)}$ Cholesterol, triglyceride and phospholipid in the liver were determined as described previously. ${ }^{15 /}$ Liver phosphatidylcholine was separated from the other lipids by thin-layer chromatography, and the fatty acid composition was determined by gas-liquid chromatography. ${ }^{16)}$ Shortchain fatty acids in the cecum were determined by gas-liquid chromatography, using SP- $1200 / 1 \% \mathrm{H}_{3} \mathrm{PO}_{4}$ on 80/100 Chromosorb. W AW (Supelco Japan Ltd., Tokyo) as the column packing materials. ${ }^{17)}$ Briefly, an aliquot of the cecum contents ( $1-2 \mathrm{~g}$ ) was homogenized by ultradispersion in acidified water ( $\mathrm{pH}$ less than 2) containing 2-ethylbutyric acid as an internal standard. The homogenate was vacuum-distilled, and the distillates were applied to the gas-liquid chromatograph.

Statistics. Data were analyzed by a one-way analysis of variance, which was followed by an inspection of all differences by Duncan's multiple range test or Student's $t$ test. ${ }^{18)}$

\section{Results}

\section{Experiment 1}

The XO diet was given to diabetic and nondiabetic rats. As shown in Fig. 1, growth was markedly suppressed by the streptozotocin treatment, but the magnitude of retardation was less severe during the course of the

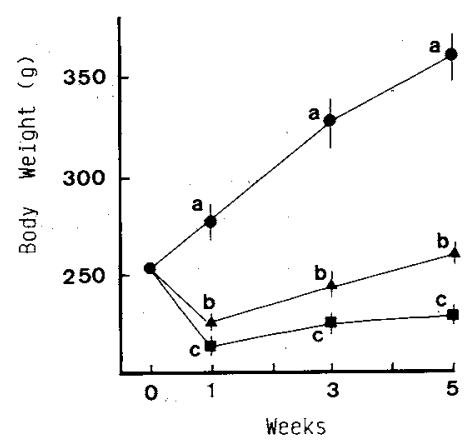

Fig. 1. Effect of Xylooligosaccharides on Growth (Exp. 1).

Diabetic rats, 8 per group, were given the basal ( $\square$ ) or the $\mathrm{XO}$ diet (A). Four non-diabetic rats were given the $\mathrm{XO}$ $\operatorname{diet}(\boldsymbol{O})$. Each point shows mean \pm S.E. ${ }^{a b}$ Different letters in the vertical direction show significant difference between the group at $p<0.05$. 
experiment in the rats fed with the XO diets than in those fed with the basal diet. The diabetic rats ate less food for the first week,
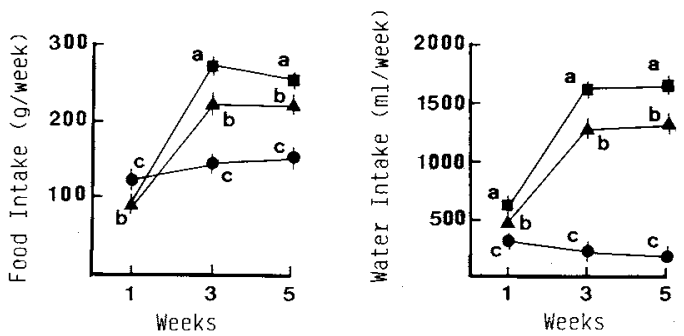

Fig. 2. Effect of Xylooligosaccharides on Food and Water Intake (Exp. 1).

Food and water intake are expressed as the values per week such as for $0-1,1-3$ and 3-5 weeks, respectively. diabetic rats fed with the basal diet; $\boldsymbol{\Delta}$, diabetic rats fed with the $\mathrm{XO}$ diet; 0 , non-diabetic rats fed with the $\mathrm{XO}$ diet. See the legend to Fig. 1. but more later on as compared to the nondiabetic rats (Fig. 2). The hyperphagia in the diabetic rats observed after the first week was significantly suppressed when the diet contained XO. Water intake was markedly high in the diabetic rats throughout the experimental period. The polydipsia due to diabetes was again less severe in the rats fed with the XO diet than in those fed with the basal diet.

As shown in Fig. 3, streptozotocin induced an elevation of the serum glucose level, but the extent was significantly less throughout the experimental period in the rats fed with the XO diet than in those fed with the basal diet. Since the blood obtained from the tail vein was too little in the nondiabetic rats after the first and the third weeks, only the cholesterol value from the blood obtained after killing after the fifth
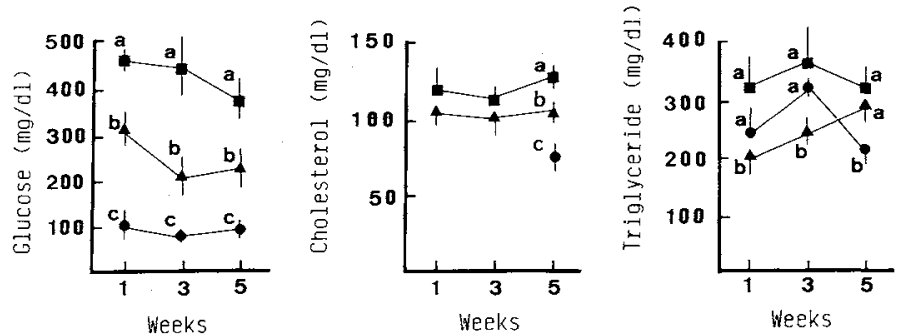

Fig. 3. Effect of Xylooligosaccharides on the Levels of Glucose, Cholesterol and Triglyceride in the Serum (Exp. 1).

$\boldsymbol{\square}$, diabetic rats fed with the basal diet; $\boldsymbol{\Delta}$, diabetic rats fed with the XO diet; $\boldsymbol{O}$, non-diabetic rats fed with the XO diet. See the legend to Fig. 1.

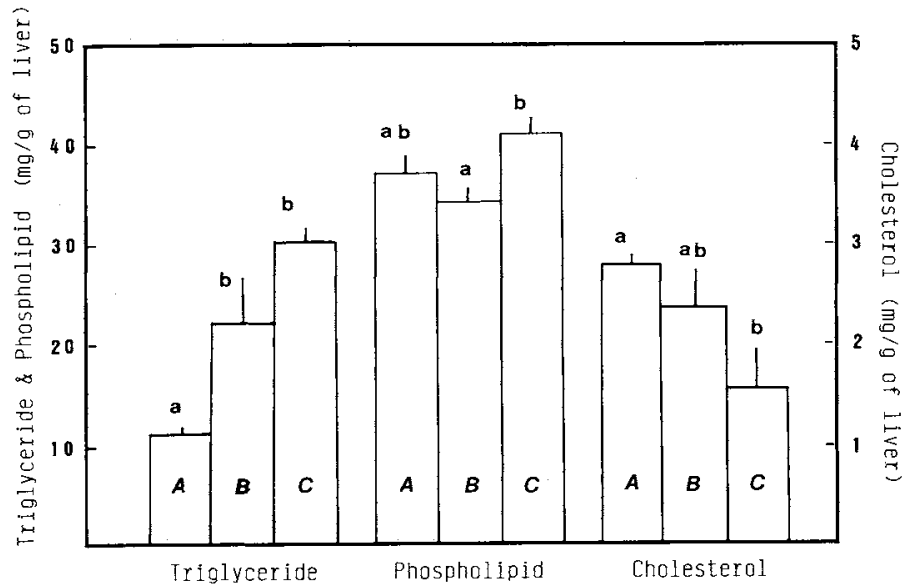

Fig. 4. Effect of Xylooligosaccharides on the Level of Hepatic Lipids (Exp. 1).

$A$, diabetic rats fed with the basal diet; $B$, diabetic rats fed with the XO diet; $C$, non-diabetic rats fed with the XO diet. See the legend to Fig. 1 . 
week is presented in Fig. 3. A significant elevation of serum cholesterol and triglyceride in the diabetic rats was observed only at the time when rats were killed. XO significantly suppressed the elevation of serum cholesterol at the time when the rats were killed. The serum triglyceride level after the first and third weeks was significantly lower in the rats fed with the $\mathrm{XO}$ diets than in those fed with the basal diet and in nondiabetic rats. At the time of sacrifice the serum triglyceride level tended to be lower in the rats fed with the XO diet than in those

Table II. EFFECT OF XYLOOLIGOSACCHARIDES ON THE FATTY ACID COMPOSITION OF Liver Phosphatidylcholine (Exp. 1)

\begin{tabular}{lcccc}
\multirow{2}{*}{$\begin{array}{c}\text { Fatty } \\
\text { acid }\end{array}$} & \multicolumn{2}{c}{ Diabetic rats } & & $\begin{array}{c}\text { Non-diabetic } \\
\text { rat }\end{array}$ \\
\cline { 2 - 3 } & Basal diet & XO diet & & XO diet \\
\hline $16: 0$ & $16.6 \pm 0.5$ & $17.6 \pm 0.4$ & & $17.2 \pm 0.5$ \\
$18: 0$ & $27.6 \pm 0.4$ & $27.5 \pm 0.5$ & & $27.6 \pm 0.4$ \\
$18: 1$ & $3.7 \pm 0.2$ & $4.2 \pm 0.1$ & & $4.0 \pm 0.2$ \\
$18: 2(n=6)$ & $15.1 \pm 1.2^{a}$ & $12.0 \pm 0.5^{b}$ & & $9.2 \pm 0.3^{c}$ \\
$20: 4(n=6)$ & $34.1 \pm 0.6^{a}$ & $36.7 \pm 0.5^{a b}$ & & $39.0 \pm 1.2^{b}$ \\
$22: 6(n=3)$ & $2.9 \pm 0.2$ & $3.0 \pm 0.2$ & & $2.9 \pm 0.2$ \\
$20: 4 / 18: 2$ & $2.37 \pm 0.23^{a}$ & $3.11 \pm 0.15^{b}$ & $4.26 \pm 0.24^{c}$
\end{tabular}

Values are mean \pm S.E. for 8 diabetic rats and 4 nondiabetic rats.

$a b$ Different superscript letters show significant difference at $p<0.05$. fed with the basal diet, but did not reach a statistically significant level.

As shown in Fig. 4, the diabetic rats fed with the basal diet had a significantly lower triglyceride level and a higher cholesterol level in the liver as compared to the non-diabetic rats. These levels in the rats fed with XO were similar to those in non-diabetic rats. The level of liver phospholipid was slightly but significantly lower in the diabetic rats fed with the XO diet than in the non-diabetic rats.

As shown in Table II, the fatty acid composition of liver phosphatidylcholine was modified by the streptozotocin treatment: an elevated proportion of linoleate was accompanied by a reduction of arachidonate in the diabetic rats, thus resulting in lower ratios of arachidonate to linoleate (a desaturation index). The extent of elevation of linoleate due to diabetes was significantly more moderate in the rats fed with the XO diet than in those fed with the basal diet. The reduction of the desaturation index, thus, was partly alleviated in the former compared with the latter.

The cecum content and the pool size of the short-chain fatty acids were measured for the diabetic rats. As shown in Fig. 5, the cecum content was more in the rats fed with the XO diet than in those fed with the basal diet. The pool size of acetic acid among the cecum short-chain fatty acids specifically increased in

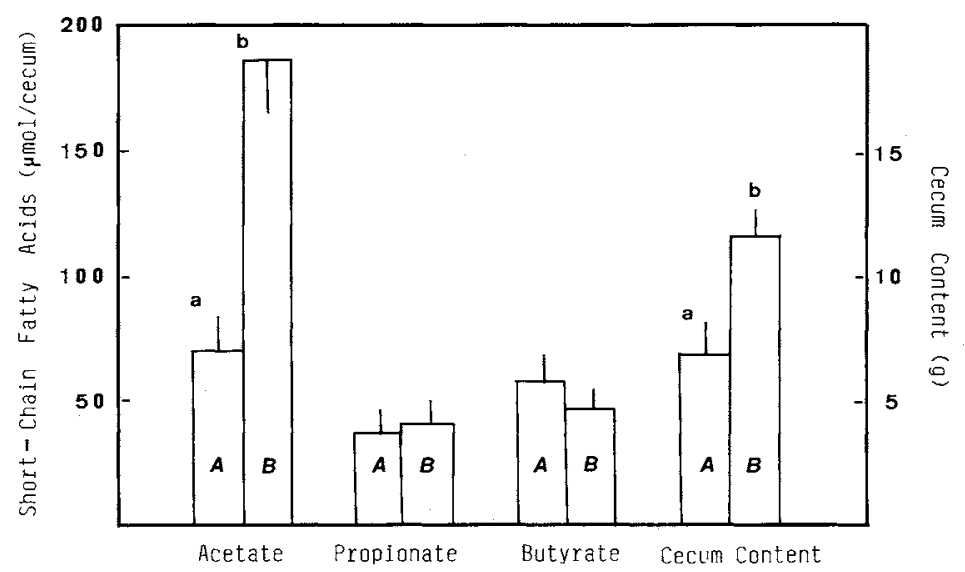

Fig. 5. Effect of Xylooligosaccharides on the Weight of the Cecum Contents and Pool Size of Short-chain Fatty Acids (Exp. 1).

A, diabetic rats fed with the basal diet; B, diabetic rats fed with the XO diet. See the legend to Fig. 1. 
Table III. EFFect of XYloOligosaccharides on THE Body Weight, FoOd Intake, SERum Glucose AND Lipids IN THE SERum AND LIVER (Exp. 2)

\begin{tabular}{lcc}
\hline Parameter & Basal diet & XO diet \\
\hline Body weight $(\mathrm{g})$ & & \\
$\quad$ Initial & $180 \pm 4$ & $180 \pm 2$ \\
Final & $237 \pm 12$ & $269 \pm 7^{*}$ \\
Food intake $(\mathrm{g})$ & $36.9 \pm 1.6$ & $32.5 \pm 0.5^{*}$ \\
Serum (mg/d) & & \\
$\quad$ Glucose & $616 \pm 19$ & $567 \pm 12^{*}$ \\
Triglyceride & $511 \pm 64$ & $404 \pm 58$ \\
$\quad$ Cholesterol & $\mathrm{n} . \mathrm{d}$. & $\mathrm{n} . \mathrm{d}$. \\
Liver (mg/g of liver) & & \\
Triglyceride & $11.2 \pm 0.8$ & $22.1 \pm 4.4^{*}$ \\
Cholesterol & $2.8 \pm 0.1$ & $2.3 \pm 0.4$ \\
\hline
\end{tabular}

Values are mean \pm S.E. for 7 rats per group. * Significantly different from the basal diet at $p<0.05$.

the rats fed with the $\mathrm{XO}$ diet, no detectable changes being observed in propionate and butyrate. The concentration ( $\mu \mathrm{mol} / \mathrm{g}$ wet weight) of butyrate was significantly lower in the rats fed with the XO diet than in those fed with the basal diet $(8.5 \pm 1.4$ for the basal diet and $4.1 \pm 0.6$ for the $\mathrm{XO}$ diet, $p<0.05$ ).

\section{Experiment 2}

In order to address the question of whether the beneficial response of the diabetic parameters just described to the $\mathrm{XO}$ diet was merely attributable to the absence of sucrose in the $\mathrm{XO}$ diet, the $\mathrm{XO}$ diet prepared by replacing $10 \%$ of corn starch in the basal diet as shown in Table I was given to the diabetic rats for 5 weeks.

As shown in Table III, the XO diet improved the body weight gain and hyperphagia, tended to decrease the level of serum glucose and triglyceride, and increased the liver triglyceride level as compared with the basal diet.

\section{Discussion}

The present results show that a supplementation of XO at the expense of carbohydrates (starch and sucrose) improved such diabetic symptoms as growth retardation, polydipsia and hyperphagia, elevation of serum glucose, cholesterol and triglyceride, reduction of liver triglyceride, and reduction of the desaturation index (ratio of arachidonate to linoleate) in the liver phosphatidylcholine.

Carbohydrate restriction has been reported to decrease the blood glucose and glucosuria in streptozotocin-induced diabetic rats. ${ }^{18)}$ According to the report by Siegel et al., however, severe carbohydrate restriction, at least from $50 \mathrm{~g} / 100 \mathrm{~g}$ of diet to $25 \mathrm{~g} / 100 \mathrm{~g}$ of diet, was necessary to achieve a sufficient improvement to growth retardation as well as serum glucose elevation in streptozotocininduced diabetic rats. $^{20}$ In the present experiment, only $10 \mathrm{~g}$ of carbohydrate $/ 100 \mathrm{~g}$ of diet was replaced with XO. Thus, it is assumed that the beneficial effects observed in the present study can be attributed to the supplementary XO rather than to the carbohydrate restriction, although the latter effect can not be totally excluded.

On the basis of the experimental result that carbohydrate restriction to the diabetic rats improved hyperphagia and the urinary loss of glucose derived from dietary carbohydrates, Siegel et al. ${ }^{20)}$ suggested that severe glucosuria causes an energy loss in the urine, and thus the rats tended to demand more energy to maintain the body weight. The beneficial effect of the XO diet on hyperphagia and probably on growth retardation may partly be attributed to the reduction of energy loss into the urine.

The XO preparation used in the present experiment was mainly composed of freexylose, xylobiose and xylotriose. The xylose in the preparation may not have any relevance to the improvement of diabetic symptoms, since xylose is absorbed by facilitated diffusion through the intestinal cells ${ }^{21}$ and is even clinically utilized as an index for the glucose absorption test. ${ }^{22)}$ Since $\mathrm{XO}$ is stable in an acidic medium in vitro, ${ }^{11}$ the oligosaccharides may not be degraded while passing through the stomach. As an analogy to xylan, which is not decomposed by the digestive enzymes in the small intestine due to the presence of a xylose polymer with a $\beta$-1-4-linkage, the appearance of free xylose derived from the oligosaccharides 
seems to be extremely unlikely in the small intestine, at all. Judging from the observations that the XO diet increased the recovery of the cecum content and the pool size of short-chain fatty acids, it can be predicted that large amounts of XO escape the digestion process in the small intestine. In fact, the XO preparation was resistant in vitro to the digestive enzymes. $^{23)}$

The present results show that the XO diet specifically increased the pool size of acetic acid in the cecum. This finding may have relevance to the amelioration of polydipsia and growth retardation for the following reasons: First, short-chain fatty acids have been shown to increase water and sodium absorption in the distal intestine, ${ }^{24)}$ thus, partly compensating for the massive water loss into the urine in the diabetic state without orally taking a large amount of water. Second, the acetic acid produced in the cecum is easily absorbed into the circulation system ${ }^{25)}$ and transferred mainly to the muscles, ${ }^{26)}$ where the acetate appears to be utilized as a source of energy. ${ }^{27)}$ Active production of acetic acid in the cecum and probably in the colon can thus alleviate the degradation of muscle protein that is used as a source of energy in the diabetic condition.

An acidic environment in the cecum and colon has been reported to be preferable for preventing the proliferation of putrefactive bacteria. ${ }^{28)}$ In the present study, the concentration of cecum butyrate, which is one of the candidates for the foul smell in the feces, was significantly lower in the rats fed with the $\mathrm{XO}$ diet than in those fed with the basal diet. In humans, an oral supplementation of $\mathrm{XO}$ has been shown to cause a lowering of fecal $\mathrm{pH}$ and an enrichment of Bifidobacterium. ${ }^{29}$ Oligosaccharides have also been shown to be selectively used by Bifidobacterium, but not by Clostridium or E. coli, in an in vitro fermentation system. ${ }^{30}$ )

The present experiment shows that the XO diet increased the liver triglyceride level almost to the level in non-diabetic rats. This phenomenon can be considered to be one of the beneficial effects of the XO diet rather than an adverse effect, although fatty liver may occur due to the overflow of free fatty acids into the liver from the adipose tissue in diabetic rats. $^{31)}$ Kudchodker et $a l .{ }^{32)}$ have recently reported that the type of dietary protein influences blood glucose and liver triglyceride levels in streptozotocin-induced diabetic rats: a reduction of the serum glucose level in rats fed with casein as compared to soybean protein was accompanied by increased lipogenesis in the liver from $\left[{ }^{14} \mathrm{C}\right]$ octanoate, suggesting an improvement to the excessive oxidation of fatty acids. In addition, a diet supplemented with fructooligosaccharides, which have been reported to improve diabetic symptoms in rats $^{13)}$ and humans, ${ }^{9)}$ also increased the liver triglyceride level in diabetic rats as compared with effect from the basal diet (unpublished observation). Thus, it is suggested that the $\mathrm{XO}$ diet given to the diabetic rats may divert the flow of fatty acids in the liver into the lipogenic pathway from the ketogenic pathway. ${ }^{31)}$

In summary, the present results show that preparations of $\mathrm{XO}$ are beneficial for improving the growth performance and several metabolic parameters in diabetic rats. The underlying mechanism(s) for these beneficial effects exerted by the XO diets should be evaluated. Furthermore, it would be worthwhile to evaluate the application of $\mathrm{XO}$ as a sweetener for diabetic patients.

\section{References}

1) H. C. Trowell, Am. J. Clin. Nutr., 25, 926 (1972).

2) D. J. A. Jenkins, A. R. Leeds, C. Newton and J. M. Cummings, Lancet, 1, 1116 (1975).

3) N. S. Painter and D. P. Burkitt, Br. Med. J., 2, 450 (1971).

4) S. A. Bingham, D. R. R. Williams and J. H, Cummings, Br. J. Cancer, 52, 399 (1985).

5) H. Hidaka, M. Hirayama and N. Sumi, Agric. Biol. Chem., 52, 1181 (1988)

6) O. Ozawa, Food Chemicals (in Japanese), 6, 75 (1988).

7) F. W. Parrish, F. B. Talley, K. D. Ross, J. Clark and J. G. Phillips, J. Food Sci., 44, 813 (1979).

8) K. Endo, M. Kumemura, M. Sugawara, Y. Tashiro, K. Nakamura, K. Suzuki, T. Fujisawa, N. Shiragami, K. Suzuki and T. Mitsuoka, in "Intestinal Flora and Bio-Homeostasis," ed. by T. Mitsuoka, Japan 
Scientific Society Press, Tokyo, 1989, p. 31.

9) K. Yamashita, K. Kawai and M. Itakura, Nutr. Res., 4, 961 (1984).

10) I. R. Crossley and R. Williams, Gut, 25, 85 (1984).

11) S. Fujikawa and M. Okazaki, Food Chemicals (in Japanese), 1, 63 (1989).

12) A. Jundo, A. E. Lambert, W. Stauffacher and A. E. Renold, J. Clin. Invest., 48, 2129 (1969).

13) Y. Hata, U. Takeda and T. Niizato, in "Reports of the Neosugar Conference" (in Japanese)," ed. by N. Hosoya, Meiji Seika Co., Tokyo, 1982, p. 63.

14) J. Folch, M. Lees and G. H. Sloane-Stanley, J. Biol. Chem., 226, 497 (1957)

15) Y. Nagata, K. Imaizumi and M. Sugano, Br. J. Nutr., 44, 113 (1980).

16) M. Sugano, K. Ryu and T. Ide, J. Lipid Res., 25, 474 (1984).

17) T. Hoverstand, O. Faust, A. Bjorneklet and T. Bohmer, Scand. J. Gastroenterol., 19, 375 (1984).

18) G. W. Snedecor and W. G. Cochran, in "Statistical Methods, 6th. ed.," Iowa State Univ. Press, Ames, IA, 1976, p. 258 and p. 298.

19) E. Siegel, V. Trapp and F. Harting, Diabetologia, 13, 432 (1977).

20) E. G. Siegel, V. E. Trapp, C. B. Wolleheim, A. E. Renold and F. H. Schmidt, Metabolism, 29, 421 (1980).

21) R. K. Crane, Fed. Proc., 24, 1000 (1965).

22) D. J. A. Jenkins, T. M. S. Wolever, A. R. Leeds, M.
A. Gassull, P. Haisman, J. Dilawari, D. V. Goff, G. L. Metz and K. G. M. M. Alberti, Br. Med. J., 1, 1392 (1978).

23) M. Okazaki, H. Koda, R. Izumi, S. Fujikawa and N. Matsumoto, J. Jpn. Soc. Nutr. Food Sci. (in Japanese), in press.

24) W. E. M. Roediger and A. Moor, Dig. Dis. Sci., 26, 100 (1981).

25) H. Ruppin, S. Bar-Meir, K. H. Soergel, C. M. Wood and M. G. Schmitt, Gastroenterology, 78, 1500 (1980).

26) S. E. Knowles, I. G. Jarrett, O. H. Filsell and F. J. Baallard, Biochem. J., 142, 401 (1974).

27) C. L. Skutches, C. P. Holroyde, R. N. Myers, P. Paul and G. A. Reichard, J. Clin. Invest., 64, 708 (1979).

28) K. Itoh, N. Shirakami and T. Mitsuoka, in "Intestinal Flora and bio-Homeostasis," ed. by T. Mitsuoka, Japan Scientific Society Press, Tokyo, 1989, p. 5.

29) M. Okazaki, S. Fujikawa and N. Matsumoto, J. Jpn. Soc. Nutr. Food Sci. (in Japanese), 43 (6) (1990).

30) M. Okazaki, S. Fujikawa and N. Matsumoto, Bifidobacteria Microflora (in Japanese), 9, 77 (1990). (1990).

31) P. Felig, in "Metabolic Control and Disease," ed. by P. K. Bondy and L. E. Rosenberg, W. B. Saunders Co., Philadelphia, PA, 1980, p. 276.

32) B. J. Kudchodkar, M.-J. C. Lee, S.-M. Lee, N. M. DiMarco and A. G. Lacko, J. Lipid Res., 29, 272 (1988). 